\title{
Efficacy of Pertussis Vaccines: A Brighter Horizon
}

\author{
N. W. PRESTON, T. N. STANBRIDGE
}

British Medical fournal, 1972, 3, 448-451

\section{General Plan and Methods}

\section{Summary}

A study of pertussis infections in 186 children under 11 years of age in the Manchester region during 1969-71 suggests that recently-manufactured vaccines have been more effective than those made before 1967. The earlier vaccines were effective mainly against the serotypes of Bordetella pertussis possessing antigen 2, while those made from 1967 are more nearly equal in their effectiveness against thedi fierent serotypes. $A$ booster dose of the earlier vaccines did not prevent infection with type 1,3 organisms, but we obtained a positive culture from only one child who had received four doses of recent vaccine.

Simultaneous infection of a child with two or more serotypes was frequently seen. The predominant serotype in a patient was usually type 1,3 ; less often it was type 1,2,3 or type 1,2; it was never type 1. A change of serotype sometimes occurs during the course of the illness and is probably directed by the vaccination status of the patient in relation to the serotype of the initial infection.

Our findings emphasize the need for vaccines to contain adequate amounts of all three pertussis agglutinogens, and for satisfactory immunization schedules to be used in their administration.

\section{Introduction}

The pertussis vaccines used in the United Kingdom in fieldtrials by the Medical Research Council $(1951,1956,1959)$ were shown to vary greatly in their protective potency. In most cases there was good correlation between their potency in children and their ability to protect mice against intracerebral challenge. The best batches of vaccine gave more than $80 \%$ protection in children at that time, and the mouse protection test has been used since then to control the potency of batches. By 1966-67, however, the protective effect of vaccination was less than $30 \%$ (Public Health Laboratory Service, 1969).

The possibility that immunity to whooping cough may be type-specific (Preston, 1963) has been reinforced by subsequent findings (Chalvardjian, 1965; Kuznetsov, 1969; Preston, 1970a; Blaskett et al., 1971). These have shown that the serotype of Bordetella pertussis that is prevalent in a community may be influenced by the antigenic composition of the vaccine in local use, and they have emphasized the need for pertussis vaccine to contain adequate amounts of all three heat-labile agglutinogens.

The validity of these conclusions has been brought into question by the discovery reported by Kuznetsov (1969) and by Zakharova and Gorbunova (1969) and confirmed by our own preliminary tests that a child may be infected with two serotypes simultaneously. The present investigation was designed to study the extent of such mixed infection, particularly in relation to the age and vaccination history of the patient.

Department of Bacteriology and Virology, University of Manchester N. W. PRESTON, M.D., F.R.C.PATH., Reader

T. N. STANBRIDGE, M.B., M.R.C.PATH., Lecturer

The whooping-cough patients from whom specimens were examined included inpatients at the Duchess of York Hospital for Babies, Manchester, and children in their own homes in or near Manchester. Many of these were in Salford or Stretford and formed a part of the continuing surveillance by the Public Health Laboratory Service which followed the survey mentioned above (Public Health Laboratory Service, 1969).

The initial pernasal swab cultures were sent to us from the Public Health Laboratory, Manchester. When possible at least 12 colonies of Bord. pertussis were subcultured from each primary plate to charcoal-blood-agar (Oxoid, 1965), and these subcultures were typed by slide agglutination as previously described (Preston, 1965a, 1970a). In a minority of cases only confluent growth was obtained on the primary plate or fewer than 12 colonies appeared.

Vaccination histories were sought from parents, general practitioners, and health departments. A child was regarded as "vaccinated" only if a written record confirmed at least three inoculations of pertussis (or triple) vaccine. "Unvaccinated" children were those for whom the parent's negative statement was supported by the absence of written records. Patients with an absent or doubtful vaccination history or with fewer than three inoculations were placed in separate groups from these two categories.

Differences between groups of children are described as significant (P 0.05) or highly significant (P 0.005) by applying the calculations of Loewenthal and Wilson (1939) given in their table of differences.

\section{Results}

Bord. pertussis was cultured from 186 children under 11 years of age in the period February 1969 to May 1971. Of these, 158 were swabbed in the eight-month period 1 June 1970 to 31 January 1971,131 of them in the second half of 1970.

As in previous studies in the U.K. over the past 10 years (Preston, 1963; Report, 1965; Public Health Laboratory Service, 1969) most of the strains isolated were of type 1,3. However, in $49(26 \%)$ of the 186 cases there was a preponderance (over half of the colonies tested from the primary culture) of colonies possessing antigen 2 (type $1,2,3$ or type 1,2 ) (Table I).

\section{EFFECTIVENESS OF VACCINATION}

There was a highly significant difference between children aged 4-10 years who would have been vaccinated with batches manufactured before 1967 and those aged 1-3 years vaccinated in 1967 or later (Table I). Out of 53 children with known vaccination history in the older group from whom Bord. pertussis was isolated $33(62 \%)$ had been vaccinated. This is about the same proportion as that of vaccinated children in the local population, and it confirms the conclusions of the Public Health Laboratory Service (1969) that these vaccines gave very little protection. In contrast only $14(27 \%)$ of the 52 bacteriologically-confirmed cases in the younger group had been vaccinated. This suggests that the recently-manufactured vaccines were more effective. Moreover, we are able to offer explanations for the failure of the vaccination in many of these 14 cases below. 
TABle I-Cases From Which Bord. Pertussis was Isolated, Listed According to History of Pertussis Vaccination. Numbers of Cases in which Cultures had Predominance of Strains possessing Antigen 2 are given in Parentheses

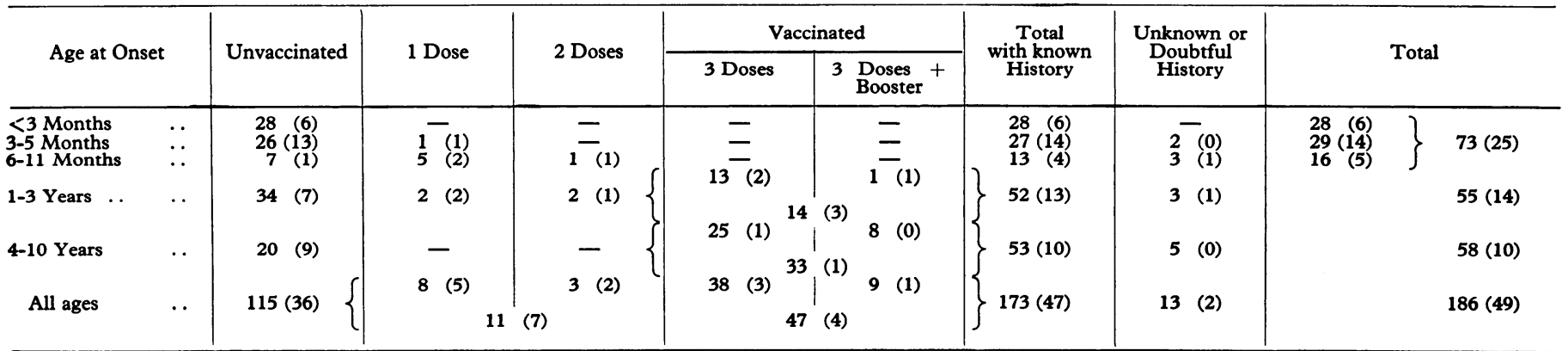

\section{VACCINATION AND SEROTYPES ISOLATED}

In children aged 4-10 years there was a highly significant difference in serotype between cultures from unvaccinated and vaccinated patients. In the unvaccinated group (Table I) nine out of 20 children had a predominance of strains possessing antigen 2 , whereas in the vaccinated group there was only one out of 33, and this child had received her first injection at only 3 weeks of age, perhaps too young to respond well to whatever antigens were present in the vaccine.

This tendency for antigen 2 to be detected in cultures from unvaccinated more than from vaccinated children is shown also by analysis of cases in which it was possible to test at least 12 colonies from each child. There were 12 such children in the unvaccinated group, and $60(42 \%)$ of the 144 colonies from them possessed antigen 2 . In contrast only $29(12 \%)$ of the 240 colonies from 20 vaccinated children possessed antigen 2 .

This confirms the often-repeated claim (Preston, 1971) that the earlier vaccines given to these older children were more effective against strains with antigen 2 than against serotype 1,3.

In children aged 1-3 years there was no significant difference in serotype between the cultures from those who had been vaccinated and those who had not (Table I), and this suggests that recently manufactured vaccines may be more nearly equal in their effectiveness against the different serotypes.

\section{CHILD'S AGE AND SEROTYPES ISOLATED}

Organisms with antigen 2 predominated in a smaller proportion of children aged $4-10$ years $(10 / 58 ; 17 \%)$ than of those under 1 year old $(25 / 73 ; 34 \%$ ) (Table I), but this reflects the influence of vaccination in the older children, as noted above. In unvaccinated children aged 4-10 years a high proportion $(9 / 20$; $45 \%$ ) had a predominance of strains possessing antigen 2 .

Indeed, the only significant difference in serotype between the strains isolated from different age groups of unvaccinated children was between those aged 3-5 months, in which $13 / 26$ $(50 \%)$ had a predominance of strains possessing antigen 2 , and those under 3 months, with $6 / 28(21 \%)$. This difference is highly significant when comparison is made between cultures from the two groups in cases where it was possible to test at least 12 colonies from each child. There were 21 such children in each group; in those aged 3-5 months $144(57 \%)$ of the 252 colonies from them possessed antigen 2, but in those aged under 3 months there were only $87(35 \%)$. This difference may possibly be due to the persistence, in infants aged under 3 months, of maternal antibody (which is usually lacking in antibody to factor 3 ), and this may have caused an increased proportion of type 1,3 strains in this group.

\section{INFLUENCE OF FOURTH (“BOOSTER”) DOSE}

Bord. pertussis was isolated from 33 children aged 4-10 years who had been vaccinated, and of these eight had received a booster dose (Table I). This is not surprising, for the Public Health Laboratory Service (1969) showed that a booster dose of vaccines made before 1967 did not reduce the attack rate. We note, also, that all eight had infections that were predominantly of serotype 1,3 .

On the other hand, among children aged 1-3 years, who would have been vaccinated in 1967 or later, positive cultures were obtained from 14 vaccinated patients, but only one had received a booster dose. Moreover, this child had a predominance of organisms possessing antigen 2 ( 9 out of 12 colonies tested), suggesting that the infection in this particular child resulted not so much from an inadequacy in antigenic content of the vaccine-for example, a lack of antigen 3-as from an overall failure of the immune response, even to the highly-antigenic factor 2 component. The implication of these findings may be that recently-manufactured vaccines are very effective when administered as a primary course of three injections followed by a booster dose but that the primary course alone may not provide adequate immunity. An assessment of the significance of these results, however, should allow also for the tendency during the past few years for a proportion of children to be vaccinated according to the schedule of the Central Health Services Council (1968), which makes no provision for a fourth dose of pertussis.

\section{INFLUENCE OF ONLY ONE OR TWO DOSES}

There were 11 children in our series (Table I) who had received only one or two doses of pertussis vaccine before their attack of whooping-cough. Of these, $7(64 \%)$ had an infection in which strains possessing antigen 2 predominated. This presents a picture similar to that seen in the unvaccinated child and noticeably different from that in children who had received three or four doses, in whom strains possessing antigen 2 rarely predominated. Although the numbers are too small to be significant the suggestion is that vaccination with fewer than three doses may not protect even against strains possessing antigen 2, an antigen that has been present in the past in an adequate concentration in most batches of vaccine. This apparent ineffectiveness of only one or two doses could not be attributed to vaccination at too young an age, for in none of the 11 cases was the first dose given at under 5 months of age.

\section{INFECTIVITY OF SEROTYPE 1}

Only one colony of the 504 that were tested from the primary cultures of 42 children aged under 6 months was of serotype 1 . Moreover, this serotype was detected in only four of the 186 children in our entire series, and in each of these four cases it accounted for only one of the colonies tested. The predominant serotype in these four children varied as shown in Table II.

From these findings we may conclude that type 1 is a degenerate form, probably resulting from antigen loss by mutation 
TABLE II-Four Children in whose Primary Cultures Serotype 1 was Detected

\begin{tabular}{|c|c|c|c|c|c|}
\hline \multicolumn{4}{|c|}{ No. of Colonies of Each Serotype } & \multirow{2}{*}{$\begin{array}{c}\text { Total No. of Colonies } \\
\text { Tested from } \\
\text { Child's Primary } \\
\text { Culture }\end{array}$} & \multirow{2}{*}{$\begin{array}{l}\text { Predominant } \\
\text { Serotype }\end{array}$} \\
\hline $\begin{array}{l}\text { Type } \\
1,2,3\end{array}$ & $\begin{array}{c}\text { Type } \\
1,2\end{array}$ & $\begin{array}{c}\text { Type } \\
1,3\end{array}$ & Type & & \\
\hline$\frac{9}{17}$ & $\begin{array}{r}2 \\
26 \\
1 \\
-\end{array}$ & $\begin{array}{r}-1 \\
28 \\
7\end{array}$ & $\begin{array}{l}1 \\
1 \\
1 \\
1\end{array}$ & $\begin{array}{r}12 \\
28 \\
47 \\
8\end{array}$ & $\begin{array}{l}1,2,3 \\
1,2 \\
1,3 \\
1,3\end{array}$ \\
\hline
\end{tabular}

(Preston, 1970b), and that it is not capable of establishing infection in a child by itself; either antigen 2 or antigen 3 or both must be present in an infecting strain.

It should be noted, in contrast, that serotype 1 can be highly virulent for mice, whereas strains freshly isolated from children (type 1,2,3, type 1,2, or type 1,3) are avirulent (Preston, 1966). Indeed a type 1 strain can be used for the challenge in the mouse protection test.

\section{CHANGE OF SEROTYPE DURING COURSE OF ILLNESS}

In this series there were 19 cases from which more than one specimen yielded Bord. pertussis. In six of these there was a change of serotype (highly significant in four of them) during the course of illness (Table III). In Cases 1-5 the colonies tested from the initial specimen were all of type 1,3 , but antigen 2 was also detected in subsequent specimens. These five children were all unvaccinated, and it seems likely that they were infected initially with the type 1,3 strains now prevalent in this country and that type 1,2,3 mutants arose during the course of the illness and, in some cases, outgrew and replaced the original serotype in an environment where there was no selective influence due to antibody.

The opposite change in serotype was noted by Kuznetsov (1969) and by Zakharova and Gorbunova (1969). The Russian vaccines in use in their areas were stated to be lacking in antigen 3 , and most of the patients investigated by Zakharova and Gorbunova had been vaccinated. In cultures taken during the first two weeks of illness most of the strains possessed antigen 2, for types 1,2,3 and 1,2 were prevalent in their localities; but in specimens taken later type 1,3 predominated. We agree with Kuznetsov in attributing this change of serotype to the presence of a relatively high titre of antibody 2 in vaccinated children, and we feel that a similar explanation is likely in Case 6. However, as this child was from a broken home and there had not been continuity of medical attention we were unable to establish the vaccination status.

In Cases 7-19 (Table IV) no significant change of serotype was shown. In most a predominance of one serotype $(1,2,3,1,2$, or 1,3) persisted, perhaps because no mutants developed in these cases. In others a stable balance of serotypes appeared to have been achieved. Perhaps Case 19 is worthy of comment, although the numbers of colonies tested on each occasion were too small to justify very significant conclusions in this case. The child had received only one dose of vaccine, which may, however, have enabled her to respond relatively rapidly to a subsequent exposure to antigen 2 (see above). The type $1,2,3$ mutants which appear to have developed from the initial 1,3 infection may therefore have been suppressed soon and the infection reverted to type 1,3 .

IABLE III-Six Cases in which Change of Serotype Occurred during Course of Illness

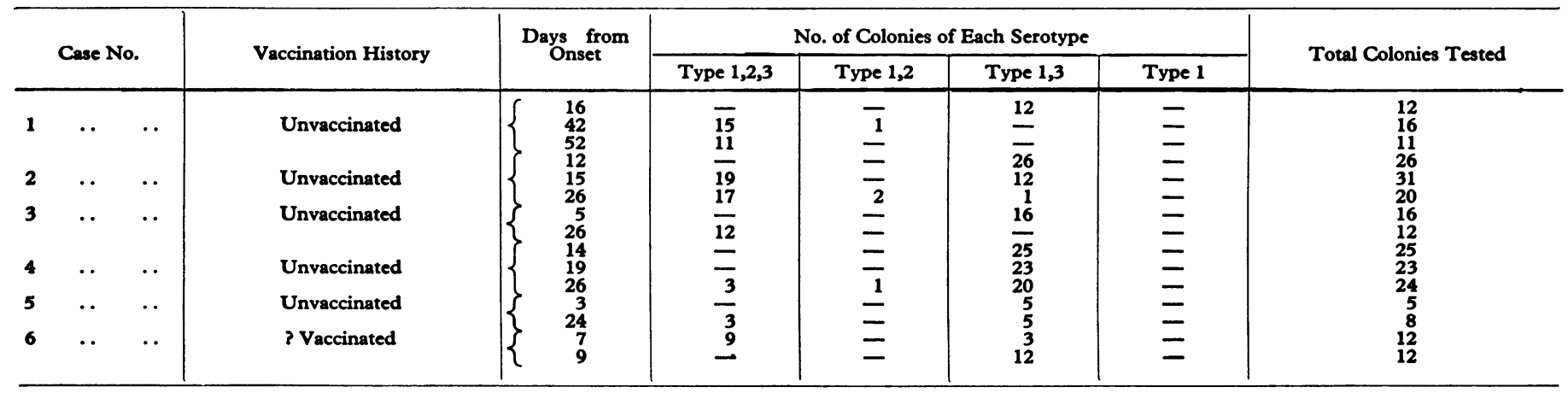

Table IV-Thirteen Cases in which the Same Serotype(s) Persisted During Course of Illness

\begin{tabular}{|c|c|c|c|c|c|c|c|c|c|}
\hline & \multirow{2}{*}{\multicolumn{2}{|c|}{ Case No. }} & \multirow{2}{*}{$\begin{array}{l}\text { Vaccination History } \\
1 \text { dose } \\
\text { Unvaccinated }\end{array}$} & \multirow{2}{*}{$\begin{array}{c}\begin{array}{c}\text { Days from } \\
\text { Onset }\end{array} \\
77 \\
21 \\
15 \\
19 \\
13 \\
16 \\
19 \\
\times \quad\end{array}$} & \multicolumn{4}{|c|}{ No. of Colonies of Each Serotype } & \multirow{2}{*}{$\frac{\text { Total Colonies Tested }}{24}$} \\
\hline & & & & & $\frac{\text { Type 1,2,3 }}{24}$ & $\frac{\text { Type } 1,2}{-}$ & $\frac{\text { Type } 1,3}{-}$ & $\frac{\text { Type } 1}{-}$ & \\
\hline $\begin{array}{l}17 \\
18\end{array}$ & $\begin{array}{l}\cdots \\
\cdots \\
\cdots \\
\cdots \\
\cdots \\
\cdots \\
\cdots \\
\cdots \\
\cdots \\
\cdots\end{array}$ & $\begin{array}{l}\cdots \\
\cdots \\
\cdots \\
. \\
\cdots\end{array}$ & $\begin{array}{l}1 \text { dose } \\
\text { Unvaccinated } \\
\text { Unvaccinated } \\
\text { Unvaccinated } \\
\text { Unvaccinated } \\
\text { Unvaccinated } \\
\text { Unvaccinated } \\
\text { ? Vaccinated } \\
1 \text { Dose } \\
\text { Unvaccinated } \\
\text { Vaccinated (3 doses) } \\
\text { Unvaccinated } \\
1 \text { dose }\end{array}$ & $\begin{array}{r}7 \\
21 \\
15 \\
19 \\
13 \\
16 \\
19 \\
\times \quad r \\
\times+7 \\
\times+13 \\
7 \\
17 \\
5 \\
15 \\
24 \\
28 \\
3 \\
7 \\
21 \\
7 \\
16 \\
5 \\
29 \\
1 \\
8 \\
10 \\
15 \\
12 \\
26 \\
40\end{array}$ & $\begin{array}{r}24 \\
24 \\
6 \\
6 \\
13 \\
11 \\
19 \\
15 \\
15 \\
14 \\
4 \\
6 \\
14 \\
12 \\
= \\
= \\
= \\
= \\
= \\
= \\
= \\
1 \\
1 \\
1 \\
1\end{array}$ & $\begin{array}{l}= \\
= \\
= \\
= \\
\overline{1} \\
= \\
\overline{2} \\
14 \\
= \\
= \\
= \\
= \\
= \\
= \\
=\end{array}$ & $\begin{array}{l}\text { 二 } \\
\overline{1} \\
1 \\
5 \\
2 \\
1 \\
1 \\
3 \\
1 \\
10 \\
10 \\
1 \\
\frac{2}{2} \\
22 \\
12 \\
22 \\
24 \\
19 \\
20 \\
12 \\
12 \\
10 \\
21 \\
11 \\
4\end{array}$ & $\begin{array}{l}= \\
= \\
= \\
= \\
= \\
= \\
= \\
= \\
= \\
= \\
= \\
= \\
= \\
=\end{array}$ & $\begin{array}{r}24 \\
24 \\
6 \\
6 \\
14 \\
16 \\
21 \\
16 \\
16 \\
15 \\
8 \\
9 \\
24 \\
22 \\
16 \\
12 \\
2 \\
22 \\
12 \\
22 \\
24 \\
19 \\
20 \\
12 \\
12 \\
10 \\
22 \\
12 \\
4 \\
5\end{array}$ \\
\hline
\end{tabular}




\section{Discussion}

Bord. pertussis was isolated from a smaller proportion of vaccinated children aged 1-3 years than vaccinated children aged 4-10 years, the difference being highly significant (Table I). This is presented as an indication of greater efficacy of vaccines manufactured during or after 1967.

It might be suggested that this difference could be atttibuted to remote vaccination in the older children, but two previous investigations (Report, 1965; Public Health Laboratory Service, 1969) showed no evidence of increasing susceptibility to pertussis infection as the interval from vaccination to exposure increased.

Another possible explanation would be a dramatic decrease in the acceptance of pertussis vaccine by the local population in recent years, for which again there is no evidence.

It seems hardly likely that practitioners would ask for pernasal specimens to be taken from vaccinated older children in preference to vaccinated younger children with paroxysmal coughs; indeed, the reverse might possibly be expected.

We are therefore led to suggest that recent vaccines have been more effective. It is true, however, that from the design of our investigation we have no evidence of lower morbidity in vaccinated children of the younger age group compared with the older group, but merely fewer isolations of Bord. pertussis. But it seems unlikely that the attack rate of clinical disease caused by Bord. pertussis would remain unaltered by vaccination while the bacteriologist's ability to establish the true nature of the illness by isolating the causative organism was noticeably reduced; and certainly this was not so with vaccines made before 1967 . Nevertheless, even if morbidity due to Bord. pertussis were not decreased in the vaccinated population one might surmise that vaccines which could cause a highly significant decrease in the isolation of the organism from the nasopharynx would also reduce the spread of bordetella from one child to another and thereby decrease the attack rate in the population.

We have shown that vaccines made before 1967 were effective against the serotypes from which they were made, being rich in antigen 2, but many batches were devoid of antigen 3 and they did not protect against serotype 1,3. This was realized by 1963 (Preston, 1963; Wilson et al., 1965), and Muggleton (1967) stated that since 1964 all manufacturers in Great Britain have included type 1,3 strains in their vaccines. Nevertheless, we were unable to detect antigen 3 in batches made in May and June 1965 (Preston, 1965b), and three vaccines made in 1967 varied in their content of antigen 3 (Abbott et al., 1971); one (X) gave a good antibody response in both children and rabbits, the second $(Y)$ gave a poor response, while antigen 3 was not detected in the third (Z). From other studies not yet complete it seems likely that much of the vaccine used in the U.K. during the past four years can stimulate a good antibody response in children to all three of these pertussis antigens, though in some antibody 3 has been detected only after a fourth (booster) dose of vaccine.

These considerations enable us to offer an explanation for the failure of recently-made vaccines to protect 14 of the present series of children aged 1-3 years (see above). In three (all with type 1,3 infections) one of the three doses of vaccine was made by manufacturer $\mathrm{Z}$ and may well have been lacking in antigen 3 . Three others received their first dose when less than 2 months old, and their response may have been restricted by either maternal antibody or immunological immaturity or both (Taylor, 1970); indeed, most (14/16) of the colonies tested from one of these children contained antigen 2, suggesting a failure to respond even to the highly antigenic factor 2 component of the vaccine. In two other cases the precise ages at vaccination were not available, and one at least of these probably received the first dose when too young.

The six remaining children appear to have had a satisfactory vaccination schedule. Four (with type 1,3 infections) received only three doses of vaccine; three were vaccinated when about 3,4 , and 6 months old, and the fourth was vaccinated according to the schedule of the Central Health Services Council (1968) at $5,6 \frac{1}{2}$, and 12 months. This suggests that for some children a fourth (booster) dose may be necessary for immunity against type 1,3 infection. The other two children had a predominance of type 1,2,3 organisms, even though one of them had received a fourth dose of vaccine, and we suggest that they may be part of the small residue of a population which can be expected to give a poor response even to a good vaccine administered according to a satisfactory schedule, and they therefore remained susceptible even to organisms possessing antigen 2 .

Lastly, we note that the 33 vaccinated children aged 4-10 years included 12 who had an apparently satisfactory schedule. In this age group they would probably have received vaccine rich in antigen 2 but deficient in antigen 3, and in none of their infections did organisms with antigen 2 predominate. Five of the 12 had received a booster, and type 1,3 strains alone were recovered from these. Our recommendation that doses of vaccine should be given according to a satisfactory schedule starting when the child is not less than 3 months old must therefore be coupled with the necessity for vaccine to contain adequate amounts of all three pertussis agglutinogens, the commonest deficiency in the U.K. being antigen 3. With acceptance of these two conditions our findings give reason to believe that pertussis vaccines will again become highly effective in the future.

We wish to thank Dr. J. D. Abbott, of the Regional Public Health Laboratory, Manchester, who isolated the cultures of Bord. pertussis throughout the period of our investigation and kindly sent them to us. We are grateful also to him and to Dr. C. L. Miller, of the Epidemiological Research Laboratory, Colindlae, through whom many of the vaccination histories were obtained, and also to many general practitioners, to the health departments of Manchester, Salford, and Stretford, and to the records office of the Duchess of York Hospital, from whom we obtained similar information about other patients.

\section{References}

Abbott, J. D., Preston, N. W., and Mackay, R. I. (1971). British Medical fournal, 1,86

Blaskett, A. C., Gulasekharam, J., and Fulton, L. C. (1971). Medical fournal of Australia, 1, 781 .

Central Health Services Council (1968). Immunization against Infectious Disease. London, Department of Health and Social Security.

Chalvardjian, N. (1965). Canadian Medical Association fournal, 92, 1114. Kuznetsov, E. A. (1969). Trudy Nauchnoi Konferencii po Epidemiologii $i$ Immunologii Koklyusha i Parakoklyusha, 1968, p. 165. Moscow.

Loewenthal, L. J. A., and Wilson, W. A. (1939). British Medical fournal, 2,110 .

Medical Research Council (1951). British Medical fournal, 1, 1463.

Medical Research Council (1956). British Medical fournal, 2, 454

Medical Research Council (1959). British Medical fournal, 1, 994

Muggleton, P. W. (1967). Public Health, 81, 252.

Oxoid Manual, (1965). 3rd edn., p. 90. London, Oxoid Ltd.

Preston, N. W. (1963). British Medical fournal, 2, 724.

Preston, N. W. (1965a). British Medical fournal 2, 11 .

Preston, N. W. (1965b). British Medical fournal, 2, 1001.

Preston, N. W. (1966). Fournal of Pathology and Bacteriology, 91, 173

Preston, N. W. (1970a). Laboratory Practice, 19, 482.

Preston, N. W. (1970b). Symposia Series in Immunobiological Standardization, vol. 13, p. 18. Basel, Karger.

Preston, N. W. (1971). In Pan American Health Organization, Washington, D.C., Scientific Publication No. 226, p. 371.

Public Health Laboratory Service Report (1969). British Medical fournal, 4, 329

Report of Public Health Laboratory, Manchester; City of Manchester Health Department; Department of Bacteriology, University of Manchester (1965). Monthly Bulletin of the Ministry of Health and the Public Health Laboratory Service, 24, 104.

Taylor, I. (1970). Health Trends, 2, 88.

Wilson, A. T., Henderson, I. R., Moore, E. J. H., and Heywood, S. N. (1965). British Medical fournal, 2, 623 .

Zakharova, M. S., and Gorbunova, Z. V. (1969). Trudy Nauchnoi Konferencii po Epidemiologii $i$ Immunologii Koklyusha $i$ Parakoklyusha, 1968 p. 170. Moscow. 